\title{
Local progression of needle tract metastases during neo-adjuvant chemotherapy after core biopsy in breast carcinoma: A case report
}

\author{
Judith Lamberigts ${ }^{1 *}$ and Karin Leunen ${ }^{2}$ \\ ${ }^{1}$ Registrar gynecology and obstetrics, AZ Turnhout, Belgium \\ ${ }^{2}$ Consultant UZ Leuven, MD AZ Sint Maarten Mechelen, Belgium
}

\begin{abstract}
Case study: A 55 y old woman presents with a cT2N0M0 triple negative breast cancer at our breast clinic. The upfront neo-adjuvant chemotherapy had to be interrupted due to clinically important needle tract metastasis (NTM), confirmed on imaging. Surgery was expedited and pathology report confirmed clear tumourload as NTM+.

Aim: We conducted a literature study to evaluate the prevalence and prognostic importance for NTM+ after breast cancer diagnosis, treated with NACT. Moreover, the causal relationship between these NTM+ and the differences in puncture techniques was investigated.

Conclusion: There seems to be a striking discordance between the (accidental) histopathologic diagnosis of NTM+ after surgery and prognosis. The malignant potential from these accidentally found NTM+ is not known, but seems not quite important, at least, when short interval between surgery and adjuvant radiotherapy is respected as defined in international guidelines. However, less is known about progression of these NTM+ during NACT and the consequences for local relapse and disease control at long term.
\end{abstract}

Biopsy technique as such is reported as less important, but vacuum assisted biopsy seems to reduce the incidence of the described NTM+.

Abbreviations: NTM+: Needle tract metastasis; EC:Epirubicincyclophosphamide; TCw: Paclitaxel-carboplatin weekly; PD: Progressive disease; TNBC: Triple negative breast carcinoma.

\section{Introduction}

Clinical examination, imaging and pre-operative histopathological investigation are the cornerstones of the diagnosis in breast cancer. This triple diagnosis is now an indispensable part of daily breast oncology practice.

The core biopsy with obtaining histological information offers several advantages for the oncologist. Firstly, unnecessary surgery is avoided by distinguishing between benign and malignant lesions. Secondly, an additional advantage is the avoidance of diagnostic and therapeutic procedures in 2 various surgical steps [1,2]. Third, one is also more likely to have tumour-free margins as the nature of surgery is adjusted [3]. Finally, knowing the receptor status, offers an additional important advantage in the context of whether or not neo-adjuvant therapy (NACT) is indicated or that this NACT offers the opportunity of breast-saving surgery $[4,5]$.

There are several ways to biopsy a breast carcinoma. The most used modalities are: FNAC (fine needle aspiration cytology), a CNB (core needle biopsy), a VACNB (vacuum-assisted core needle biopsy) or an excisional biopsy [6-11]. Since the beginning of puncture diagnostics in breast carcinoma, there has been concern about the possibility of spreading malignant cells along the puncture route [12-20]. Highlighting the difference in the histopathological demonsration of tumoral spread across the puncture site and the clinical relevance in the context of tumoral relapse and survival is extremely important.
In this case, our patient was a clear candidate for NACT due to histological findings (TNBC) and tumour volume (cT2 in order to achieve breast saving surgery post chemotherapy). Regular imaging during the NACT is mandatory for evaluation of the tumour response. In this case, moreover, it was this imaging that discovered the NTM+ and gave rise to a change of treatment policy during the NACT.

\section{Case}

A 55-year-old lady with a blank history was diagnosed with a breast carcinoma in the upper inner quandrant of the left breast following auto-palpation. Further investigation led to the diagnosis of a stage cT2N0M0, triple negative breast cancer with a high Ki $67>30 \%$. The initial tumor had a maximum sagittal diameter of $3.6 \mathrm{~cm}$ (Figures 1 and 2). Because of the tumor size and the fact that it was a triple negative tumor, neo-adjuvant chemotherapy in the form of epirubicin - cyclophosphamide (EC) 4 cycles every 2 weeks, normally followed by 12 cycles paclitaxel - carboplatin-weekly (TCw) was initiated.

After 4 cycles of EC and after 6 cycles TCw an increase in tumor volume was documented, although there was a clear response at

${ }^{*}$ Correspondence to: Judith Lamberigts, Registrar gynecology and obstetrics, AZ Turnhout, Putsesteenweg 163/12, 2820 Bonheiden, Belgium, Tel: 0032486734959; E-mail: judithlamberigts@hotmail.com

Key words: triple negative breast carcinoma, needle tract metastases, neo-adjuvant chemotherapy, local progression, histopathologically

Received: December 07, 2020; Accepted: December 14, 2020; Published: December 21, 2020 
radiologic evaluation after the first 3 courses of TCw. Ultrasound examination after 6 cycles TC weekly, noticed 2 additional small nodules between the skin and the edge of the tumor (Figures 3 and 4).

In order to be able to make the differential diagnosis between fat necrosis at an old puncture site or metastases at the puncture site that grew very fast, additional biopsies were taken after a new MRI. Needle tract metastases were confirmed histopathologically (Figure 5). Because of local progressive disease during neo-adjuvant chemotherapy, a new check-up for distant metastatic disease was performed and was found reassuring. Breast conserving surgery with sentinel node procedure was performed. The sentinel nodes were tumor free. The initial tumor with a size of $2.3 \mathrm{~cm}$ and 3 additional satellite lesions of $4 \mathrm{~mm}$ were retained. The morphology of the satellite lesions was identical to that of the primary breast tumor (Table 1).

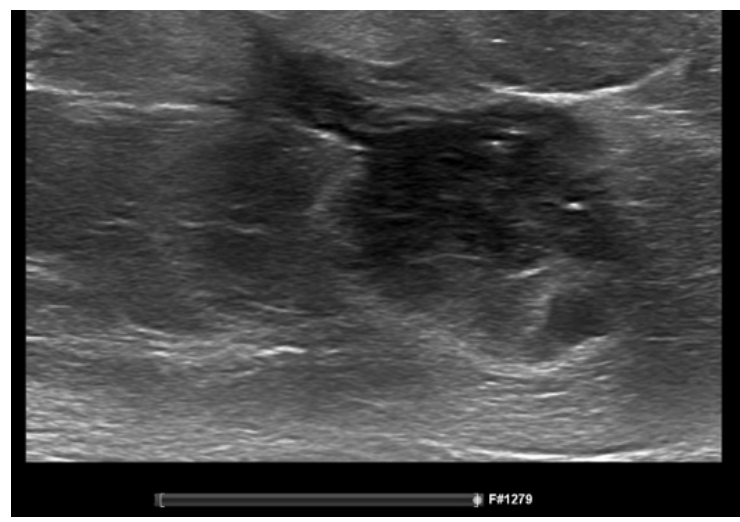

Figure 1. Ultrasound with needle tract at diagnosis
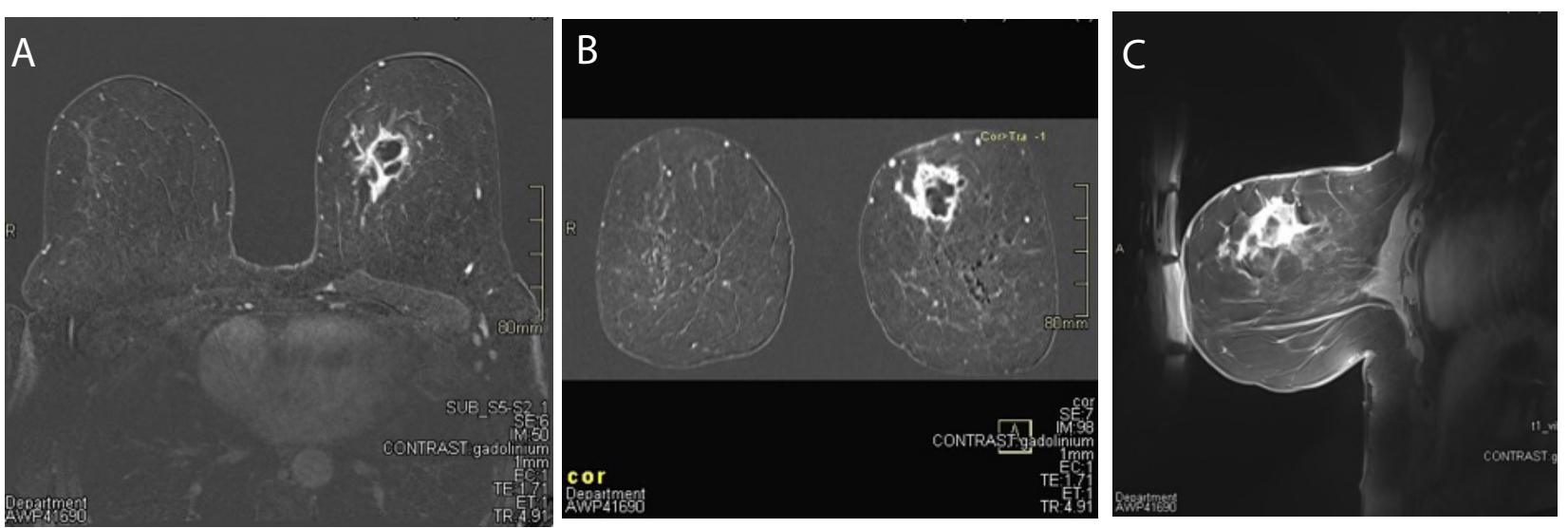

Figure 2 (A, B, C). A. MRI Transverse section at diagnosis; B. MRI coronal section at diagnosis; C.MRI sagittal section at diagnosis

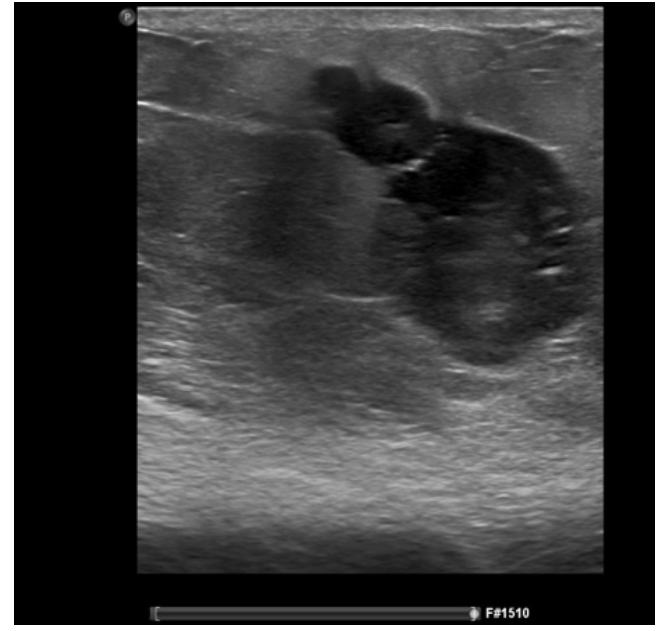

Figure 3. Ultrasound after neo-adjuvant chemotherapy with needle tract metastases 

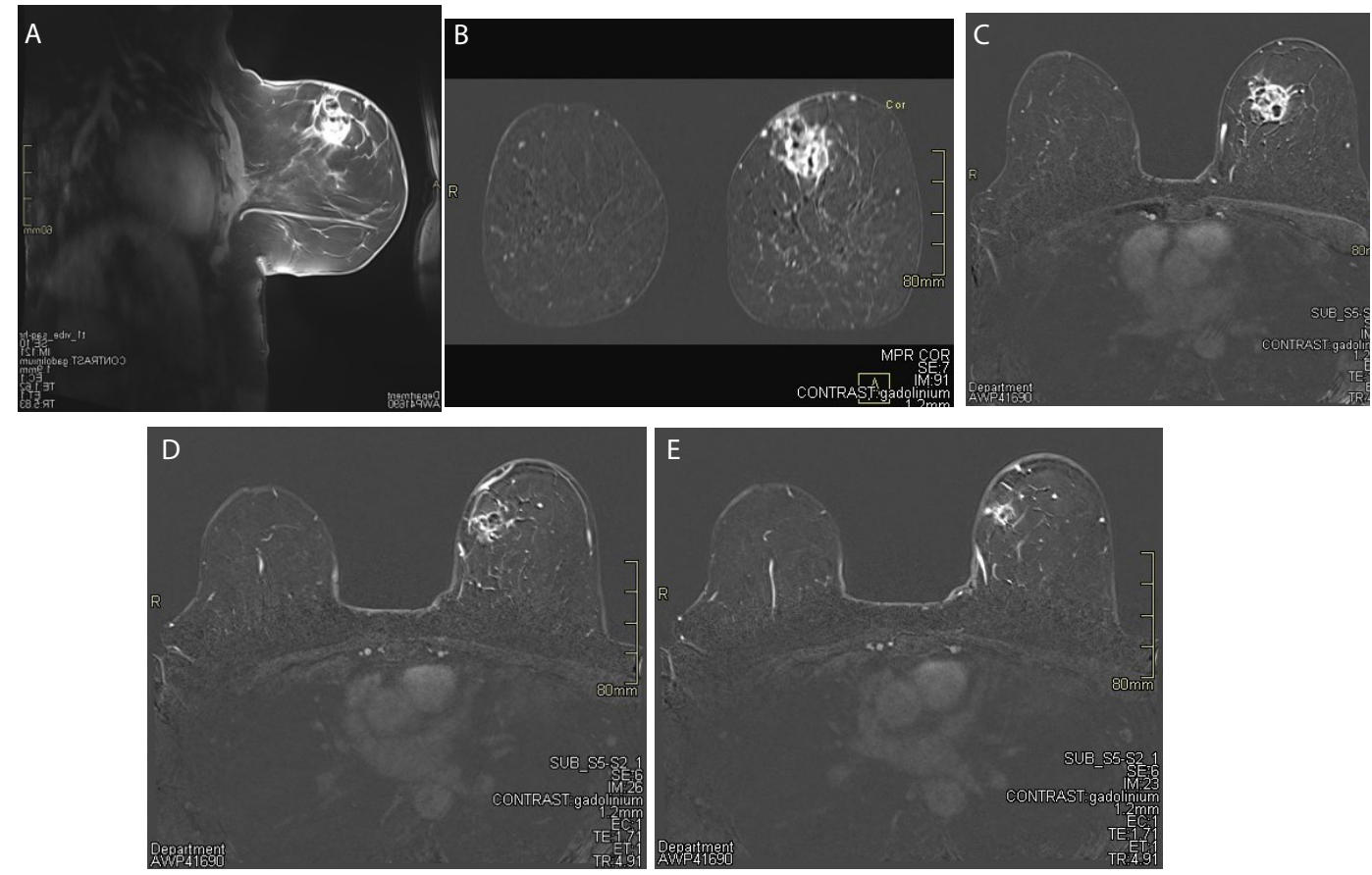

Figure 4. A. MRI sagittal section after NACT; B. MRI coronal section after NACT; C. MRI transverse section after NACT; D. MRI Large satellite nodule; E. MRI Small satellite nodule

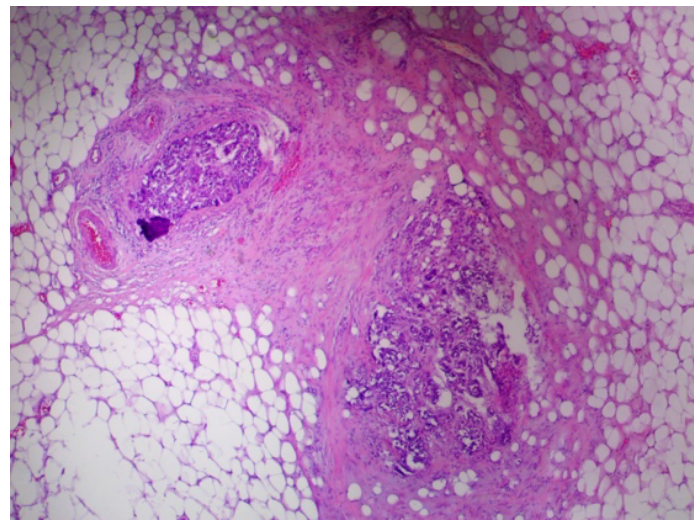

Figure 5. Histopathological examination reveals 2 small tumorous nodules in the fibrous tissue of the needle tract

Table 1. Radiological evolution of tumor dimensions

\begin{tabular}{|c|c|c|c|}
\hline & Dimensions ultrasound & Dimensions MRI & Additional findings \\
\hline Start chemotherapy & $2.6 \times 3 \mathrm{~cm}$ & $6.31 \times 3.43 \times 35.7 \mathrm{~cm}$ & \\
\hline After 4 cycles EC & $3.7 \times 3.6 \times 2.6 \mathrm{~cm}$ & & \\
\hline After 3 cycles TC & $2.5 \times 2.9 \times 1.9 \mathrm{~cm}$ & & Hypervascularization peripheral in lesion \\
\hline After 6 cycles TC & $2.8 \times 2.7 \times 2.3 \mathrm{~cm}$ & $2.6 \times 2.9 \mathrm{~cm}$ & $\begin{array}{l}\text {-Ultrasound: } 3 \text { new additional lesions of respectively } 1.1 \mathrm{~cm}, 0.4 \mathrm{~cm} \text { and } 0.3 \mathrm{~cm} \\
\text {-MRI: elongation of tumor towards skin of } 12 \mathrm{~mm} \text { and } 2 \mathrm{smaller} \mathrm{nodular} \text { components of } 4 \mathrm{~mm}\end{array}$ \\
\hline
\end{tabular}

\section{Discussion}

In their literature review Loughran et al. [6] indicate that in onethird of the biopsies needle tract seeding occurs in breast cancer patients. They emphasise that most of the displaced cells don't survive after displacement and that local tumour recurrence as a consequence of the biopsy is rare. Most of the literature about needle tract seeding does not include patients who receive upfront therapy prior to surgery. That is why, our case raises several questions about the prevalence and prognostic importance for NTM+ after breast cancer diagnosis treated with NACT. We will summarize a few elements about this topic based on our literature review. First of all we will have a look at whether a puncture biopsy poses a risk of spreading tumour cells along the puncture route and whether one biopsy technique is superior to the other (in preventing this).

Diaz et al. [21] evaluated 352 excisions in patients who first underwent a puncture biopsy and compared 3 puncture techniques (automated technique, vacuum assisted, and palpation guided). In $32 \%$ of the cases, tumour cell spread was found (114/352). They distinguished between minimal and multiple spread. They found no statistically significant difference in tumour spread between vacuum 
assisted techniques and classical puncture biopsies. $\left(\mathrm{X}^{2}=3.42, \mathrm{p}>0.05\right)$ Both invasive and non-invasive carcinomas were studied. More tumour spread was observed in invasive carcinomas than with in situ carcinomas, however, this difference was not significant. $\left(\mathrm{X}^{2}=3.58\right.$, $\mathrm{p}>0.05$ )

There was statitistically significantly more chance of tumoral spread in ductal carcinoma than in lobular or other histological subtypes. $\left(\mathrm{X}^{2}=8,000, \mathrm{p}=0.05\right)$ Tumour size or degree of differentiation showed no correlation to tumoral spread.

They also studied the time interval between biopsy and surgical excision. The larger this interval, the fewer biopsy pathways and tumoral spread could be identified. $\left(\mathrm{X}^{2}=10.768, \mathrm{p}<0.005\right)$ This suggests that the malignant potential of the tumoral spread seems relatively small. They suggest less tumoral dispersion with the vacuum assisted technique and attribute this difference to the fact that one has a lower number of puncture pathways with the vacuum technique than with the repetitive passages in a classical puncture biopsy. However, the difference is not significant.

Loughran et al. [6] could not demonstrate in their review a statistically significant difference between tumoral spread after classical core needle biopsy and fine needle aspiration. They also noted a trend towards less tumoral spread after vacuum assisted biopsies. Hoorntje et al. [22] found tumoral spread in 7 of the 13 cases studied and highlighted the possibility of excision of the biopsy tract at final surgery. They do not routinely recommend this in breast-conserving surgery as the use of adjuvant radiotherapy may be sufficient to destroy residual tumoral cells, similar to the mesothelioma [23].

A second important question, apart from whether there is local tumour spread after puncture biopsy, is the difference in risk of local relapse and survival. In other words: if these spread cells have any malignant potential?

Fitzal et al. [24] could not identify a statistically significant difference in local relapse and survival between patients who first received a puncture biopsy and those who did not. They put forward 3 possible explanations to explain the difference between puncture tract spread and the clinical local relapse ratio. Adjuvant radiotherapy, the possibility of non-viability of malignant cells after displacement and the surgical resection of the puncture tract at final surgery are 3 possible explanations.

They advise to avoid multiple punctures and recommend the use of 14-gauge needles or smaller. Kong et al. [25] found in their observational study with 3416 patients a worse survival in patients diagnosed by biopsy than in those diagnosed by local excision. They suggest a role for hematogenic spreading after biopsy. This study does not consider the indication of whether or not to perform a puncture biopsy or an excision. This may partly explain their contradictory result.

Thirdly, we looked for similar cases to ours where NTM+ occurred during NACT. There were only few data.

We found that Yam et al. [26] studied the risk of needle tract seeding in a series of patients with a TNBC (triple negative breast carcinoma) during anthracycline based NACT. 1.4\% (2/144) of their patients were found to have NTM+ and were progressive after 2 cycles of anthracyclines during ultrasound follow-up. In their study patients with inadequate response to front-line NACT underwent additional biopsies of the index lesion. They conclude that the rate of NTM+ after biopsy is low but further investigation about the long term outcomes of serial biopsies in TNBC should be investigated.
However, data concerning NTM+ during NACT and the effect of these on prognosis have not yet been investigated extensively to our knowledge. Finally, it is noteworthy that our studied patient for some time complained of moisture loss at the insertion point of the biopsy needle at the level of the skin. Whether this can be a predictive factor for needle tract metastasis is still unclear, as is its clinical relevance towards survival and local relapse.

\section{Conclusion}

It seems clear that the presence of NTM+ does not necessarily reflect a higher risk for local relapse or long-term survival disadvantages. Taking all the different aspects into account, the advantages of a histological diagnosis at the start of the treatment, seem to overcome the possible minimal disadvantages of needle tract metastases [24].

Moreover, the importance of post-operative radiotherapy and its influence on study results cannot be denied. Post-operative radiotherapy may be one of the main explanatory factors why no relevant difference is found when it comes to local relapse and long-term survival outcomes after biopsy of the initial tumor [24]. This contrasts with the fact that needle tract metastases can be demonstrated quite frequently on histopathologic examination [22].

Attention is still required when the biopsy takes place in a different quadrant as the tumorectomy. Additional resection of the puncture site/ traject can be considered in these cases. In any case, sufficient clinical information is essential for the pathologist [24].

Diaz et al. studied the relationship between the time interval of the puncture biopsy and the final surgery. The larger this interval was, the less chance there was of histopathological confirmation of needle tract metastases.

However, it is striking that this is not applicable in our case. It concerns a large time interval of 161 days because of the NACT. Despite this longer time interval, the needle tract metastases have remained viable and have not been cleared by the immune system, not even by the NACT. Tumour characteristics such as triple negativity, poorly differentiated tumours and high mitotic index could be an explanation. It would be interesting to examine these factors in a larger group of patients to evaluate the influence of NACT in the development of $\mathrm{NTM}+$. The long-term outcomes of TNBC patients receiving NACT with NTM+ should be investigated, given the results of the article of Yam et al.

Triple diagnosis remains extremely important and cannot be excluded from daily breast oncology practice. This case does not make us doubt the multiple benefits of a biopsy before therapy, but we must remain alert for the different described implications of a puncture biopsy, especially in patients receiving NACT. Avoiding multiple puncture trajects and considering vacuum assisted techniques should, in our opinion, be considered if this is not at the expense of the diagnostic information provided by a puncture biopsy. Furthermore, the importance of interim imaging in neo-adjuvant chemotherapy is again emphasized and allows for early planning of surgery when chemotherapy does not provide an additional benefit.

\section{References}

1. White RR, Halperin TJ, Olson JA Jr, SOO MS, Bentkey RC, et al. (2001) Impact of core-neelde breast biopsy on the surgical management of mammographic abnormalities. Ann Surg 233: 769-777. [Crossref]

2. King TA, Cederbom GJ, Champaign JL, Smetherman DH, Bolton JS, et al. (1998) A core breast biopsy diagnosis of invasive carcinoma allows for definitive surgical treatment planning. Am K Surg 176: 497-501. [Crossref] 
3. Wazer DE, DiPetrillo, T Schmidt-Ullrich R, Weld L, Smith TJ, et al. (1992) Factors influencing cosmetic outcome and complication risk after conservative surgery and radiotherapie for early-stage breast carcinoma. Clin Oncol 10: 356-363.

4. Spivack B, Khanna MM, Tafra L, Juillard G, Giuliano AE (1994) Margin status and local recurrence after breast-conserving surgery. Arch Surg 129: 952-957. [Crossref]

5. Van der Hage JA, van de Velde CJ, Julien JP, Tubiana-Hulin M, Vandervelden C, et al. (2001) Preoperative chemotherapy in primary operable breast cancer: Results from the European Organizaton for Research and Treatment of Cancer trial 10902. J Clin Oncol 19: 4224-4237.

6. Loughran CF, Keeling CR (2011) Seeding of tumour cells following breast biopsy: a literature review. Br J Radiol 84: 869-874. [Crossref]

7. Berg WA, Krebs TL, Campassi C (1997) Stereotactic breast biopsy: Comparison of 14 and 11-gauge directional, vacuum-assisted biopsy probes and 14-gauge biopsy guns in a breast parenchymal model. Radiol 205: 203-208.

8. Janes RH, Boutin MS (1994) Initial 300 consecutive stereotactic core-needle breast biopsies by a surgical group. Am J Surg 168: 533-537.

9. Kopans DB, Gallagher WJ, Swann CA, McCarthy KA, White G, et al. (1998) Does preoperative needle localization lead to an increase in local breast cancer recurrence? Radiol 167: 667-668. [Crossref]

10. Meyer JE, Smith DN, Lester SC, DiPiro PJ, Denison CM, et al. (1998) Large-needle core biopsy: Nonmalignant breast abnormalities evaluated with surgical excision or repeat core biopsy. Radiol 206: 716-720. [Crossref]

11. Seoudi H, Mortier J, Basile R, Curletti E (1998) Stereotactic core needle biopsy of nonpalpable breast lesions: initial experience with a promising technique. Arch Surg 133: 336-372. [Crossref]

12. Roussel F, Dalion J (1989) The risk of tumoral seeding in needle biopsies. Acta Cytol 39: 936-938.

13. Fajardo LL (1988) Breast tumor seeding along localization guide wire tracks. Radiol 169: $580-581$

14. Grabau DA, Anderson JA, Gaverson HP, Dyneberg U (1993) Needle biopsy of breas cancer: appearance of tumor cell along the needla track. Eur J Surg Oncol 19: 826-827. [Crossref]
15. Harter LP, Swengrow CJ, Panto G, Craig PH (1992) Malignant seeding of the needle track during stereotaxic core needle biopsy. Radiol 185: 713-714. [Crossref]

16. Lee KC, Chan JKC, Ho LC (1994) Histologic changes in the breast after fine-needle aspiration. Am J Surg Pathol 18: 1039-1047. [Crossref]

17. Liberman L, Vuolo M, Dershaw DD, Morris EA, Abramson AF, et al. (1999) Epithelial displacement after stereotactic 11-gauge directional vacuum-assisted breast biopsy. AJR Am J Roentgenol 172: 677-681. [Crossref]

18. Youngson BJ, Cranor M, Rosen PP (1994) Epithelial displacement in surgical breas specimens following needle procedures. Am J Surg Pathol 18: 896-903. [Crossref]

19. Youngson BJ, Liberman L, Rosen PP (1995) Displacement of carcinomatous epithelium in surgical breast specimens following stereotaxic core biopsy. Am J Clin Pathol 103: 596-602. [Crossref]

20. Berg JW, Robbins GF (1962) A late look at the safety of aspiration biopsy. Cancer 15 : 826-827. [Crossref]

21. Diaz LK, Wiley EL, Venta LA (1999) Are malignant cells displaced by large-gauge needle core biopsy of the breast? AJR Am J Roentgenol 173: 1303-1313. [Crossref]

22. Hoorntje LE, Schipper MEI, Kaya A, Verkooijen HM, Klinkenbijl JG, et al. (2004) Tumour cell displacement after 14G breast biopsy. Eur J Surg Oncol 30: 520-525. [Crossref]

23. Boutin C, Rey F, Viallat JR (1995) Prevention of malignant seeding after invasive diagnostic procedures in patients with pleural mesothelioma. A randomised trial of local radiotherapie. Chest 108: 754-758. [Crossref]

24. Fitzal F, Sporn EP, Draxler W, Mittlböck M, Taucher S, et al. (2006) Preoperative core needle biopsy does not increase local recurrence rate in breast cancer patients. Breast Cancer Res Treat 97: 9. [Crossref]

25. Kong YC, Bhoo-Pathy N, O'Rorke M, Subramaniam S, Bhoo-Pathy NT, et al. (2020) The association between methods of biopsy and survival following breast cancer: A hospital registrybased cohort study. Medicine 99: e19093.

26. Yam C, Santiago L, Candelaria RP, Adrada BE, Rauch GM, et al. Abstract P6-03-05: Risk of needle-track seeding with serial ultrasound guided biopsies in triple negative breast cancer. Cancer Res P6: 03-05.

Copyright: (C2020 Lamberigts J. This is an open-access article distributed under the terms of the Creative Commons Attribution License, which permits unrestricted use, distribution, and reproduction in any medium, provided the original author and source are credited. 